\title{
DNA damage and its repair in lymphocytes and thyroid nodule cells during radioiodine therapy in patients with hyperthyroidism
}

\author{
W Grzesiuk, J Nieminuszczy ${ }^{1}$, M Kruszewski², T Iwanienko², M Płazińska ${ }^{3}$, M Bogdańska $^{4}$, \\ E Bar-Andziak, L Królicki ${ }^{3}$ and E Grzesiuk ${ }^{1}$ \\ Department of Internal Medicine and Endocrinology, University Medical School, Warsaw, Poland \\ ${ }^{1}$ Department of Molecular Biology, Institute of Biochemistry and Biophysics, Polish Academy of Sciences, Warsaw, Poland \\ ${ }^{2}$ Department of Radiobiology and Health Protection, Institute of Nuclear Chemistry and Technology, Warsaw, Poland \\ ${ }^{3}$ Department of Nuclear Medicine, ${ }^{4}$ Department of Pathology, University Medical School, Warsaw, Poland \\ (Requests for offprints should be addressed to E Grzesiuk who is now at Institute of Biochemistry and Biophysics PAS, Pawinskiego 5a, 02-106 Warszawa, Poland; \\ Email: elag@ ibb.waw.pl)
}

\begin{abstract}
Radioiodine treatment of hyperthyroid patients with autonomous thyroid nodule leads to cellular DNA damage not only in thyrocytes but also in peripheral blood lymphocytes. The purpose of this study was to evaluate DNA breakage and base damage in thyrocytes and lymphocytes in patients treated with 131-I. In all the patients thyroid scintiscan was performed using 131-I. Damage to DNA was estimated by comet assay. Samples were taken before radioiodine treatment, and 12 and 54 days afterwards. Our results indicate high diversity in the level of DNA damage among the individual patients. However, in all cases, after 54 days the level of DNA damage in lymphocytes was similar or even lower than that in the controls. In contrast, in hot nodule the DNA damage persisted until the 54th day after 131-I application. Differences in the type of DNA damage between thyrocytes and lymphocytes were also observed. In lymphocytes there was more base damage, whereas in thyrocytes single strand breaks prevailed. This may indicate different mechanisms of DNA damage induction and/or DNA repair.
\end{abstract}

Journal of Molecular Endocrinology (2006) 37, 527-532

\section{Introduction}

Changes in thyroid function are a common cause of endocrine illness. Overproduction of thyroid hormones, hyperthyroidism, can be due to Grave's disease or autonomous thyroid nodule. In Poland, the frequency of both diseases is similar and equals $0 \cdot 14$ and $0 \cdot 18 \%$ of the population respectively. Radioiodine therapy (oral administration of 131-iodine (131-I)) is one of the most common treatments for adults with autonomous thyroid nodule, especially a hot nodule. It is effective, safe and avoids serious surgery and complications of general anesthesia. This is especially important as most patients are above the age of 50 and often suffer from cardiovascular diseases. The disadvantages include the risk of hypothyroidism and exposure to radiation dose affecting normal tissues (Ferrari et al. 1999).

The rationale for the use of 131-I in thyroid therapy is its rapid and complete absorption by the thyroid gland. After ingestion, iodine is readily absorbed from the gastrointestinal tract into the bloodstream. Upon entering the bloodstream, $30 \%$ is deposited in the thyroid, $20 \%$ is quickly excreted in feces and urine, and the remainder is gradually eliminated from the body, mainly with sweat. Clearance from the thyroid is age dependent, with biological half-lives ranging from about 10 days in infants to 80 days in adults (Elte et al. 1983, Harbert 1987, Greenspan 2001, Kramer et al. 2002). It has been found that apart from iodine, lithium may also influence thyroid functioning in euthyroidism and hyperthyroidism. It seems that the effect of lithium on the thyroid gland is similar to that of iodine. The $\mathrm{Li}^{+}$ ions inhibit thyroglobulin proteolysis, which subsequently releases thyroid hormones into circulating blood and increases the half-life of radioiodine. Lithium carbonate also acts by inhibiting the conversion of thyroxine to triiodothyronine. Due to these features, lithium carbonate is used to improve 131-I therapy in patients showing poor initial uptake of 131-I (Turner et al. 1976, Lombardi et al. 1993, Płazińska 2001).

Thyroid follicular cells accumulate iodine anions against concentration gradient with the use of sodium iodine symporter. The iodine radioisotope 131-I emits gamma $(0.38 \mathrm{MeV}$ photons $)$ and beta $(0 \cdot 19 \mathrm{MeV}$ electrons) radiation and its half-life equals 8.04 days. Emission of gamma radiation by the isotope serves to 
diagnose thyroid dysfunction, whereas beta radiation, which causes destruction of thyroid cells, constitutes the basis of thyroid disease treatment. In cells, beta particles emitted by 131-I have a range of only 1-2 mm, which is enough to irradiate all the thyroid cells accumulating iodine but not the surrounding tissues, especially the parathyroid glands (Nauman 1991, Dremier et al. 1996, Hall 2000).

Low-LET (linear energy transfer) ionizing radiation, like gamma rays or high-energy electrons, affects cells by depositing energy in DNA molecules, and causing direct DNA damage, as well as by depositing energy in the vicinity of DNA, causing water radiolysis and generating free radicals (Sonntag 1991). The thyroid gland is highly sensitive to ionizing radiation. The sensitivity is due to accumulation of free radicals in the intracellular milieu that, in consequence, disturbs several metabolic processes in follicular cells, and, most importantly, to DNA damage leading to increased level of mutations, apoptosis, necrosis, and inhibition of mitosis. The 131-I treatment causes structural changes in thyroid cells, namely vacuolization, atrophy, nuclear polymorphism, and fibrosis. However, in spite of obvious beneficial effects of treatment of hyperthyroidism with 131-I, and the cure or relief from the disease, the patients entering radioiodine therapy suffer from exposure to the radionuclide and potential damage to lymphocytes (Suwinski \& Gawkowska-Suwinska 2001).

The purpose of this study was to evaluate the potential effects of 131-I treatment, visualized as DNA breakage and base damage in thyroid cells and lymphocytes of patients with autonomous thyroid nodule. The detection of DNA damage was performed with the use of single-cell gel electrophoresis, a sensitive microscopic method allowing for detection of DNA damage at the level of individual cells.

\section{Materials and methods}

\section{Patients}

A group of 32 patients, 23 women and 9 men, aged 3780 (the mean age was 63 years) were enrolled in the study. All suffered from hyperthyroidism with autonomous thyroid nodules diagnosed by thyroid scintiscan. Patients had not been pharmacologically treated before radioiodine administration. However, patients with low 131-I uptake were given lithium carbonate at $250 \mathrm{mg}$ twice per day in a course of 10 days, 3 days prior to and 6 days after radioiodine treatment.

Fine needle aspiratory biopsy (FNA) was performed in all patients to exclude thyroid cancer. The FNAs were performed using ultrasound guidance to ensure placement of the needle in the hot nodule. All FNAs were confirmed to be diagnostic.
The protocol was submitted to and approved by the local ethical committee. All patients gave their written informed consent.

\section{Thyroid scan}

Thyroid scintiscan was performed using $0 \cdot 1 \mathrm{mCi}$ of $131-\mathrm{I}$ sodium iodide (gamma camera - Gamma MB 9420). In all cases, the thyroid scan showed a single hot nodule with suppressed radioactive iodine uptake in the remaining thyroid tissue. Nodule area was estimated by a gamma camera's computer program (Gamma Vision, Varicam, Elscint, Haifa, Israel).

\section{1-I uptake}

Directly before radioiodine treatment, 24-h uptake of 131-I was determined in all patients after oral ingestion of $50 \mu \mathrm{Ci} 131-\mathrm{I}$. The mean uptake was $28 \cdot 9 \pm 12 \cdot 2 \%$.

\section{1-I treatment}

The dose of administered 131-I was chosen according to the weight of nodule and 24-h uptake of 131-I. Thyroid nodule mass was estimated from the planimetric surface on iodine scintigram according to the formula: nodule weight $=0 \cdot 86 \times$ area of the nodule's scan. Isotope activities from 8 to $17 \mathrm{mCi}$, with average an dose of $12.48 \pm$ $1.83 \mathrm{mCi}$ were used. These doses were calculated at a minimal level to protect the extranodular tissues and minimize the risk of hypothyreosis after treatment.

\section{The effective half-life parameter}

Since in some patients, the biologic half-life $\left(\mathrm{T}^{1} / 2\right.$ biol) of intrathyroidal iodine is shortened, it is better to estimate the effective half-life ( $\left.\mathrm{T}^{1} / 2 \mathrm{eff}\right)$. This parameter is determined by the following equation:

$\mathrm{T}^{1} 1 / 2$ eff $=\left(\mathrm{T}^{1} 12\right.$ biol $\times \mathrm{T}^{1} / 2$ phys $):\left(\mathrm{T}^{1} / 2\right.$ biol $+\mathrm{T}^{1} 1 / 2$ phys $)$

$\mathrm{T}^{1} / 2$ eff - effective half-life

$\mathrm{T} 1 / 2$ biol - biological half-life

$\mathrm{T}^{1} / 2$ phys - physical half-life

\section{Comet assay}

For comet assay, two types of samples taken from patients described in Table 1, were used: peripheral blood lymphocytes (PBL) and tissue from hot nodule obtained by fine needle aspiratory biopsy. Samples were taken three times: before radioiodine treatment, and 12 and 54 days afterwards. DNA damage was assessed by the alkaline comet assay which estimates DNA strand lesions, such as single strand breaks (ssb), double strand breaks, and alkali labile sites; additionally, alkaline comet assay 
Table 1 Study characteristics of investigated patient group

Number of patients

$\begin{array}{lr}\text { Medical data } & \\ \text { Age (years) } & \\ 40-61 & 11 \\ 62-80 & 24 \\ \text { Sex } & \\ \text { F } & 26 \\ \text { M } & 9 \\ \text { Smoking } & \\ \text { Yes } & 8 \\ \text { No } & 27 \\ \text { lodine uptake (\%) } & \\ \text { Below 20 } & 6 \\ 20-40 & 23 \\ \text { Over 40 } & 6 \\ \text { Lithium treatment } & \\ \text { Yes } & 9 \\ \text { No } & 26 \\ 131-I \text { dose (mCi) } & \\ 8 & 1 \\ 12 & 30 \\ 15 & 1 \\ 17 & 3 \\ \text { T1 } 1 / 2 \text { eff } & \\ 5 \cdot 5-6 \cdot 5 & 27 \\ 6 \cdot 6-7 \cdot 5 & \end{array}$

modified by the use of DNA glycosylase (FPG) was used to estimate oxidation of DNA bases. The primary substrate for FPG is 7,8-dihydro-8-oxoguanine (8oxoG), one of the most frequently occurring base modifications in human DNA.

The alkaline comet assay was performed according to the standard protocol described in detail in Wojewódzka et al. (1998). The FPG modified comet assay was described in Kruszewski et al. (1998). In brief, microscope slides were precoated with $100 \mu$ lagarose $(0.5 \%$ Agarose Type IA (Sigma), in redistilled water) and dried. Thirty microliters whole blood or $10 \mu \mathrm{l}$ frozen lymphocytes were added to $1 \mathrm{ml}$ RPMI 1640 medium (Sigma) containing $10 \%$ FCS (Sigma) and incubated for $2.5 \mathrm{~h}$ (in the dark at room temperature). About $2-5 \times 10^{4}$ cells suspended in $100 \mu \mathrm{l}$ of $1 \%$ LMP agarose (Type VII, Sigma) in PBS were cast on precoated slides and allowed to set on ice. Once agarose was set, slides were lysed in $2 \cdot 5 \mathrm{M} \mathrm{NaCl}, 100 \mathrm{mM}$ EDTA, $10 \mathrm{mM}$ Tris, $1 \%$ Triton X-100 (Sigma), $\mathrm{pH}=10$ for $1 \mathrm{~h}$ at $4{ }^{\circ} \mathrm{C}$, in the dark. Slides for FPG treatment were washed $3 \times 5 \mathrm{~min}$ with Hepes (40 mM Hepes, $0 \cdot 1 \mathrm{M} \mathrm{KCl}, 0.5 \mathrm{mM}$ EDTA, $0 \cdot 2 \mathrm{mg} / \mathrm{ml}$ $\mathrm{BSA}, \mathrm{pH}=8$ ), at $4{ }^{\circ} \mathrm{C}$. Then, $50 \mu \mathrm{l}$ diluted enzyme was added dropwise on the agarose layer and incubated for 30 min at $37{ }^{\circ} \mathrm{C}$ in a humid chamber. During this manipulation, slides for ssb estimation were kept in the lysis buffer.

Slides were then placed in electrophoresis apparatus in electrophoresis buffer (300 mM NaOH, 1 mM EDTA,
Sigma) and left for $40 \mathrm{~min}$ at $10{ }^{\circ} \mathrm{C}$, for DNA unwinding. Electrophoresis was then carried out for $30 \mathrm{~min}$ at $1 \cdot 2 \mathrm{~V} / \mathrm{cm}$. All steps of slide preparation and electrophoresis were conducted under red light to avoid induction of additional DNA damage.

After electrophoresis, the slides were washed three times in neutralizing buffer $(0 \cdot 4 \mathrm{M}$ Tris, Sigma, $\mathrm{pH}=$ $7 \cdot 5)$, dried from the excess buffer and stained with $1 \mu \mathrm{M}$ DAPI (Sigma). Slides were left at $4{ }^{\circ} \mathrm{C}$ in humid chamber overnight and scored. Every sample was done in duplicate, 75 comets were scored per sample.

Comets were scored with computer aided image analysis system Comet v.3.0 (Kinetic Imaging Ltd, Liverpool, UK). Tail moment was taken as a measure of DNA damage.

\section{Statistical analysis}

Significance of differences between means was analysed by Student's $t$-test for dependent samples.

\section{Results}

Characteristics of all blood and thyroid tissue donors are given in Table 1. Lithium carbonate supplementation applied in some patients (Table 1) was without any influence on 131-I uptake.

Lymphocytes and thyrocytes from hot nodule taken from patients before and 12 and 54 days after 131-I treatment, were analyzed with comet assay. Our results indicate a high diversity in the level of DNA damage among individual patients even in the samples taken before 131-I application. Tail moments of control lymphocytes and thyrocytes ranged between 13 and $137 \mu \mathrm{m}$ and 10 and $132 \mu \mathrm{m}$ respectively. High diversity was also observed in DNA repair behavior. Some patients repaired DNA damage very quickly, whereas others displayed delayed repair. Interestingly, in some cases 54 days after radioiodine therapy the level of 131-I induced damage to DNA in thyrocytes and lymphocytes was even lower than in the untreated controls (data not shown). This is probably the effect of stimulation of DNA repair processes. We also observed the differences in the type of 131-I induced damage between thyroid cells and lymphocytes. In lymphocytes 12 days after iodine application $96 \%$ of patients showed a higher level of base damage than ssb. After 54 days only $76 \%$ patients showed a higher level of base damage. In thyrocytes the percentage of patients showing higher base damage 12 and 54 days after iodine application was similar (77 and $71.5 \%$ respectively, data not shown). These data indicate either more efficient DNA repair processes in lymphocytes or de novo damage formation.

Twelve days after iodine treatment, a marked increase in the level of total DNA damage (sum of ssb and 
FPG-sensitive sites) was observed (Fig. 1). However, pairwise comparison indicated that this increase was not significant, likely due to high interindividual diversity. In spite of diversity among individuals, 131-I treatment induced a statistically significant increase in DNA ssb in thyrocytes when measured 12 days after iodine application $(P=0 \cdot 02)$. A significantly $(P=0 \cdot 04)$ higher level of DNA ssb in thyrocytes was seen only 54 days after iodine treatment (Fig. 2). Interestingly, iodine treatment did not induce any significant increase in the level of base damage in thyrocytes, measured 12 or 54 days after treatment, most likely due to the high base damage in the control untreated cells (Fig. 2). No significant difference in the level of ssb or base damage in DNA was found in lymphocytes when control untreated cells were compared with those taken from the same patient 12 and 54 days after treatment (Fig. 3).

\section{Discussion}

Radioiodine therapy is a commonly used treatment for individuals with the autonomous thyroid nodule, especially the hot nodule. However, patients treated with the radionuclide may suffer from radiation induced damage to normal tissues, e.g. blood cells. The purpose of this study was to evaluate DNA breakage and base damage in thyrocytes and lymphocytes taken from 131-I treated patients with hyperthyroidism and autonomous thyroid nodule.

Statistical analysis showed a lack of correlation between $\mathrm{T}^{1} / 2$ eff, 131-I uptake, as well as iodine dose (Table 1). In spite of similar doses (most patients received $12 \mathrm{mCi}$ 131-I) the level of DNA damage in thyrocytes and lymphocytes differed significantly in individual patients. This individual variability was also appreciable in control samples (before 131-I application). The high diversity in DNA damage, which can be explained by differences in

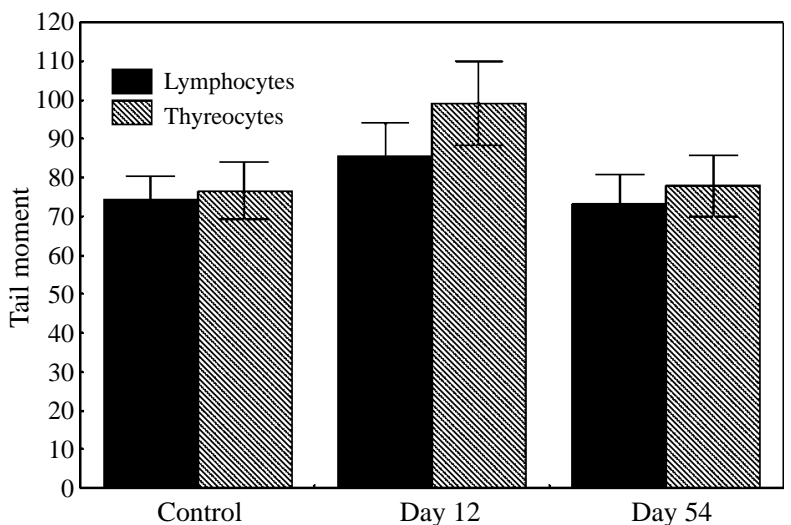

Figure 1 Effect of 131-I treatment measured as total DNA damage (sum of ssb and FPG-sensitive sites) in lymphocytes (closed bars) and thyrocytes (open bars) 12 and 56 days after radionuclide application.

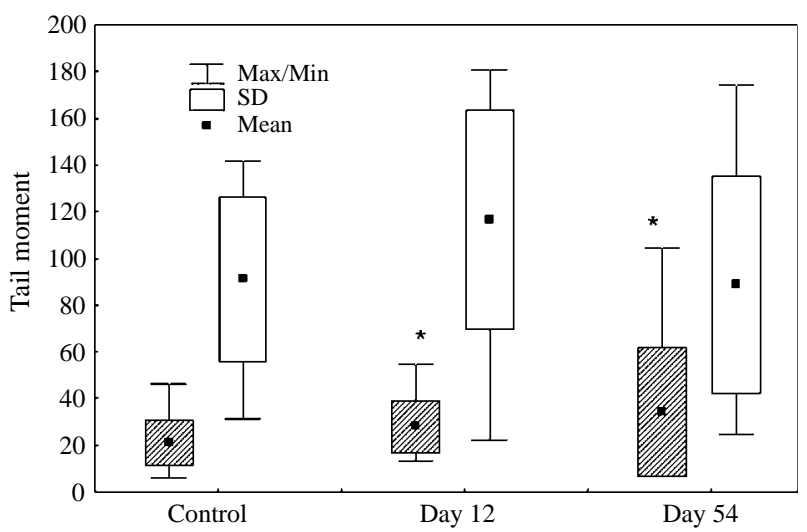

Figure 2 Radionuclide induced DNA damage to thyrocytes from patients treated with 131-I. Closed bars - single strand breaks; open bars - base damage. Asterisk denotes statistically significant difference vs control, $t$-test for dependent samples, $P<0.05$.

everyday environmental and/or occupational exposure of individual patients to genotoxic agents, was also observed by other investigators (Møller et al. 2000). However, the most important factor that influences the extent of damage to DNA seems to be the cellular 131-I uptake: the higher the 131-I uptake, the higher the DNA damage to thyrocytes. Surprisingly, no correlation was found between 131-I uptake and the level of ssb or DNA base damage in the samples taken after 131-I treatment. This points to the importance of DNA repair mechanisms in the maintenance of the steady state level of DNA damage in living cells and, in consequence, of cell survival. Since decay of 131-I is a stochastic process, a higher 131-I uptake should result in a larger number of DNA lesions. Indeed, this is true in frozen cells, where no repair processes take place (LeMotte \& Little 1984). However, in living cells the repair processes interfere with accumulation of DNA damage and modulate the geno- and cytotoxic effect of 131-I treatment. Thus, it should be recommended that patients with more efficient

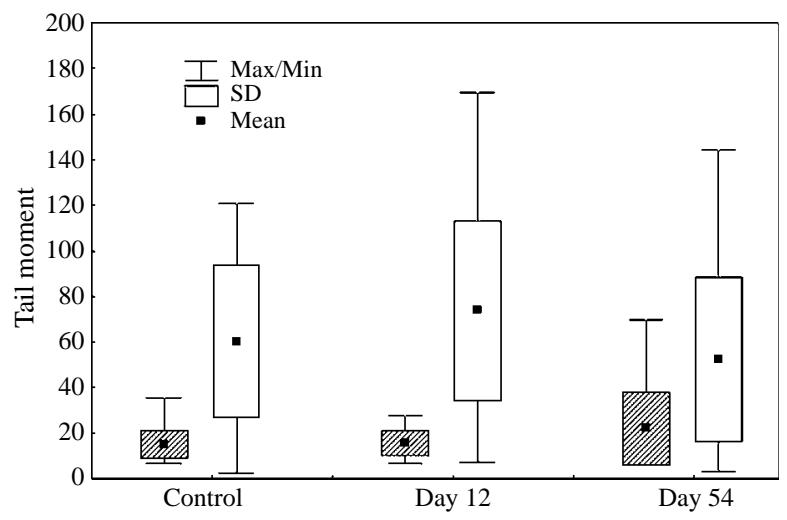

Figure 3 Radionuclide induced DNA damage to lymphocytes from patients treated with 131-I. Closed bars - single strand breaks; open bars - base damage. 
DNA repair receive higher radionuclide doses to achieve a similar therapeutic effect.

The analysis of DNA damage in thyrocytes showed significantly higher level of ssb 12 days after 131-I treatment than in the untreated controls; presumably, this increase was induced by 131-I treatment. The elevated level of ssb in thyreocyte DNA was still observed even 54 days after 131-I application (Fig. 2). Interestingly, in lymphocytes the level of ssb after treatment was comparable to that before it. Assuming a similar initial exposure of lymphocytes and thyrocytes to the bolus radionuclide dose, but shorter time of exposure of lymphocytes, due to the rapid 131-I uptake from the blood stream by thyroid nodule and fast body clearance of the remaining iodine, it seems that either lymphocytes have a more efficient DNA repair system or the persistence of radionuclide in thyrocytes disturbs the balance between DNA lesion induction and repair. The former is rather unlikely since it was reported that rejoining of DNA breaks in unstimulated human lymphocytes is reduced due to the low intracellular deoxyribonucleotides pools (Green et al. 1994). Hence, the latter seems to be more probable, as chronic exposure to low doses of ionizing radiation was reported to result in a decreased DNA repair capacity (Plappert $e t$ al. 1997).

In this work, we also applied the modified version of comet assay to assess DNA base damage, using FPG for damage recognition. FPG is a multifunctional enzyme that shows several activities, the most important being the DNA glycosylase activity that removes modified purines from DNA. Our results indicate that in contrast to ssb, 12 days after 131-I treatment the level of base damage is similar in thyrocytes and lymphocytes. This might indicate that impairment of DNA repair system due to the continuous exposure to 131-I emitted ionizing radiation affects base excision repair (BER) to a lesser extent than ssb rejoining. On the other hand, it is well known that DNA break rejoining is the final step of BER. This discrepancy can be explained by the fact that although DNA break rejoining is the final step of BER, it is not necessary for base removal. Thus, oxidized bases can be removed without subsequent strand rejoining or with delayed strand rejoining. If that is the case, it will result in a decrease in the level of FPG sensitive sites in DNA and the simultaneous increase in DNA strand breaks. This is confirmed by impaired repair of locally multiple-damaged sites (LMDS). LMDS is a multiple DNA lesion containing different types of DNA damage, such as oxidized bases, sites of base loss (apyrimidinic/ apurinic sites) and single-strand breaks (Ward 2000). LMDS are more difficult to repair than 'single' lesions; furthermore, secondary lesions, such as DNA breaks, may be formed as a result of their repair (Blaisdell et al. 2001).

In summary, we observed that in 131-I treated patients the level of DNA damage is lower in PBL than in thyrocytes. We propose that this may be a result of the less efficient DNA repair system in thyrocytes, possibly caused by the chronic exposure to the radionuclide-generated ionizing radiation. However, it cannot be excluded that accumulation of radionuclide in thyrocytes also contributes to the higher level of DNA damage in these cells. Further study is necessary to confirm these assumptions. The low level of DNA damage observed in lymphocytes indicates that lymphocytes are not a target for 131-I and the observed fluctuations in the level of damage to DNA in these cells are not 131-I-dependent.

\section{Acknowledgements}

The authors declare that there is no conflict of interest that would prejudice the impartiality of this scientific work.

\section{References}

Blaisdell JO, Harrison L \& Wallace SS 2001 Base excision repair processing of radiation-induced clustered DNA lesions. Radiation Protection Dosimetry 97 25-31.

Dremier S, Coppee F, Delange F, Vassart G, Dumont JE \& Van Sande J 1996 Thyroid autonomy: mechanism and clinical effects. Journal of Clinical Endocrinology and Metabolism 81 4187-4193.

Elte JW, Bussemaker JK, Termorshuizen W, Goslings BM \& Roelfema F 1983 Iodine kinetics in patients with euthyroid multinodular goiter compared with normal subjects. Acta Endocrinologica 3 307-312.

Ferrari C, Reschini E \& Paracchi A 1999 Treatment of the autonomous thyroid nodule. European Journal of Endocrinology 135 383-390.

Green MH, Waugh AP, Lowe JE, Harcourt SA, Cole J \& Arlett CF 1994 Effect of deoxyribonucleosides on the hypersensitivity of human peripheral blood lymphocytes to UV-B and UV-C irradiation. Mutation Research 315 25-32.

Greenspan FS 2001 The thyroid gland. In Basic and Clinical Endocrinology, 7 edn, pp 215-294. Eds FS Greenspan \& DG Gardner. New York: Lange Medical Books.

Hall EJ 2000 Doses and risk in diagnostic radiology, interventional radiology and cardiology and nuclear medicine. In Radiobiology for the Radiologist. pp 226-227. Philadelphia: Lippincott Williams and Wilkins.

Harbert JC 1987 Nuclear Medicine Therapy, pp 1-36. New York: Thieme Medical Publisher.

Kramer GH, Hauck BMM \& Chamberlain MJ 2002 Biological half-life of iodine in adults with intact thyroid function and in athyreotic persons. Radiation Protection Dosimetry 102 129-135.

Kruszewski M, Wojewódzka M, Iwanienko T, Collins AR \& Szumiel I 1998 Application of the comet assay for monitoring DNA damage in workers exposed to chronic low dose irradiation. II. Base damage. Mutation Research 416 37-57.

LeMotte PK \& Little JB 1984 DNA damage induced in human diploid cells by decay of incorporated radionuclides. Cancer Research $\mathbf{4 4}$ 1337-1342.

Lombardi G, Panza N, Biondi B \& Di Lorenzo L 1993 Effects of lithium treatment on hypothalamic-pituitary-thyroid axis: a longitudinal study. Journal of Endocrinological Investigation 16 259-263.

Møller P, Knudsen LE, Loft S \& Wallin H 2000 The comet assay as a rapid test in biomonitoring occupational exposure to DNAdamaging agents and effect of confounding factors. Cancer Epidemiology, Biomarkers and Prevention 9 1005-1015. 
Nauman J 1991C Czarnobyl, iodine, thyroid. Polish Journal of Endocrinology 42 3-14.

Plappert UG, Stocker B, Fender H \& Fliedner TM 1997 Changes in the repair capacity of blood cells as a biomarker for chronic low-dose exposure to ionizing radiation. Environmental and Molecular Mutagenesis 30 153-160.

Płazińska MT 2001 The effect of lithium carbonate on 131-I therapy in patients with hyperthyreoidism. PhD Thesis, Medical University of Warsaw, Warszawa.

Sonntag C 1991 The chemistry of free-radical-mediated DNA damage. Basic Life Sciences 58 287-317.

Suwinski R \& Gawkowska-Suwinska M 2001 Radiobiologic basis for using 131-I to treat patients with thyroid cancer. Wiadomości Lekarskie $1266-277$.
Turner JG, Browline BEW \& Rogers TGH 1976 Lithium as an adjunct to radioiodine therapy for thyrotoxicosis. Lancet $1614-615$.

Ward JF 2000 Complexity of damage produced by ionizing radiation. Cold Spring Harbor Symposia on Quantitative Biology 65 377-382.

Wojewódzka M, Kruszewski M, Iwanienko T, Collins AR \& Szumiel I 1998 Application of the comet assay for monitoring DNA damage in workers exposed to chronic low dose irradiation. I. Total damage. Mutation Research 416 21-35.

Received 21 September 2006 Accepted 25 September 2006 